\title{
Modernización ecológica y sociedad del riesgo
}

\section{Hacia un análisis de las relaciones entre ciencia, medio ambiente y sociedad}

\section{Christian Oltra}

Universitat de Barcelona. Departament de Sociologia i Anàlisi de les Organitzacions Av. Diagonal, 690. 08034 Barcelona

christianoltra@yahoo.es

\section{Resumen}

En este artículo se pretende avanzar en la comprensión de la relación entre sociedad y medio ambiente y en el papel de la ciencia en la crisis medioambiental. A partir de la confrontación de dos perspectivas teóricas de la sociología - la modernización ecológica y la teoría de la sociedad del riesgo_-, se desarrolla una visión sobre el papel que la ciencia y la tecnología desempeñan en el modo como las sociedades avanzadas hacen frente a los problemas medioambientales. La perspectiva de la modernización ecológica y la teoría de la sociedad del riesgo ofrecen una visión enfrentada sobre los procesos de cambio social derivados de la crisis. La comparación de ambas perspectivas intenta aportar elementos teóricos útiles para el desarrollo de la sociología medioambiental y para la comprensión del proceso de reforma medioambiental de la sociedad.

Palabras clave: modernización ecológica, sociedad del riesgo, ciencia, medio ambiente.

Abstract. Ecological modernization and risk society. Towards the analysis of science, environment and society

In this article we try to advance in the understanding of the relation between society and the environment and in the role of science in the environmental crisis. Through the confrontation of two theoretical perspectives from sociology - the ecological modernization theory and the theory of risk society-, we develop a vision of the role of science and technology in the way advanced societies face environmental problems. The perspective of the ecological modernization and the theory of the risk society offer a faced vision on the process of social change derived from the crisis. The comparison of both perspectives tries to contribute to the theoretical development of the environmental sociology and to the understanding of the process of environmental reform in society.

Key words: ecological modernization, risk society, science, environment. 


\section{Sumario}

Introducción La ciencia en la perspectiva

La relación entre el medio ambiente de la modernización ecológica

y el conocimiento científico Conclusiones

El papel de la ciencia en la teoría de la sociedad del riesgo

Bibliografía

\section{Introducción}

La ciencia es una de las instituciones sociales fundamentales de las sociedades modernas. Desde la primera modernidad, los avances científicos la convierten en una actividad capital en las sociedades, al tiempo que la racionalidad científica se inserta en el centro de la cultura moderna. El flujo arrollador de descubrimientos científicos que tiene lugar en el siglo XX en Estados Unidos, Europa y Japón convierten a dicha actividad en un elemento clave en el terreno económico, político y cultural de las sociedades avanzadas. Sin embargo, la crisis ambiental, reflejo de la creciente insostenibilidad de la relación entre las sociedades modernas y el medio ambiente, plantea numerosas cuestiones sobre el papel de la ciencia en la sociedad. La crisis ambiental, cuyos efectos se constatan de manera alarmante a partir de la segunda mitad del siglo XX, cuando la degradación del medio ambiente adquiere dimensiones globales, hunde sus raíces en el desarrollo de la modernidad. La crisis ambiental es, también, una crisis social de las instituciones en las que se fundamenta la sociedad moderna. La crisis plantea una reconsideración de las prácticas institucionalizadas que la han producido y, por lo tanto, una reconsideración del papel de la ciencia en la sociedad.

La constatación de la crisis medioambiental ha propiciado en la sociología la búsqueda de bases teóricas a partir de las cuales lograr una mejor comprensión de los procesos de cambio social derivados de la crisis. El papel de la ciencia y la tecnología en este proceso de cambio social ha recibido especial atención desde la sociología. Distintas perspectivas teóricas han incidido sobre las transformaciones sociales derivadas de la crisis, dando diferentes visiones sobre el cambio en la relación entre ciencia, medio ambiente y sociedad. El presente trabajo pretende analizar dos perspectivas de gran relevancia teórica para la sociología: la teoría de la sociedad del riesgo y la teoría de la modernización ecológica. Ambas perspectivas comparten su interés por el análisis del cambio social en las sociedades industriales avanzadas, pero difieren en numerosos aspectos. La modernización ecológica y la teoría de la sociedad del riesgo poseen una visión muy diferente, si no enfrentada, de la ciencia y su papel en el cambio social. En las páginas que siguen, se intenta desarrollar una aproximación a la ciencia y el medio ambiente a partir de ambas perspectivas, de modo que sea posible lograr una comprensión más adecuada del papel de la ciencia en el desarrollo de una sociedad sostenible. 


\section{La relación entre el medio ambiente y el conocimiento científico}

El medio ambiente es, en gran medida, dominio del conocimiento científico. La ciencia sitúa su objeto de estudio en la naturaleza y, a su vez, actúa sobre la misma al permitir, a través de la tecnología, la adaptación de las sociedades a su medio ambiente. La ciencia es un elemento clave en la relación de una sociedad con su medio ambiente. El modo de adaptación de una sociedad al ecosistema depende, en gran medida, de su tecnología, su conocimiento científico y de las relaciones sociales en que éstos se insertan. Así lo han puesto de manifiesto las formulaciones teóricas de la ecología humana y de la sociología medioambiental, que han concedido una importancia fundamental a la tecnología, variable considerada esencial en el estudio de la relación entre sociedad y medio ambiente junto con la organización social y la población (Martell, 1994). La dimensión científico-tecnológica de los daños medioambientales es puesta de manifiesto a partir de la segunda mitad del siglo XX, cuando los peligros para la supervivencia de la humanidad del avance tecnológico descontrolado son divulgados en obras como Science and Survival (Commoner, 1966). Los posibles efectos de la ciencia y la tecnología sobre el medio ambiente alcanzan una magnitud dramática con los accidentes nucleares de Three Mile Island y Chernobyl, que se convierten en símbolos de la vulnerabilidad de la tecnología nuclear, pero, también, de la pérdida de autoridad del consejo científico en la toma de decisiones y de la pérdida de credibilidad de los políticos que confían en él (Weingart, 1999).

El papel de la ciencia en la relación entre la sociedad y el medio ambiente cambia de modo sustancial con la crisis medioambiental. La ciencia pasa de estar orientada al dominio y al control de la naturaleza, papel que tradicionalmente ha ocupado en la cultura occidental, a la gestión, ajuste y adaptación de la naturaleza (Funtowicz y Ravetz, 1999). El problema, ahora, no es la conquista de la naturaleza, sino evitar la destrucción y degradación de la misma. Por este motivo, cada vez es mayor el énfasis por parte de políticos, grupos de interés y ciudadanos en una política ambiental fundamentada en un preciso conocimiento científico de los problemas del medio. Se piensa que las decisiones sobre la solución de los problemas medioambientales serán más acertadas si están basadas en información científica objetiva (Steel y otros, 2003). El conocimiento científico legitima, así, las decisiones políticas, que requieren, cada vez más, de este conocimiento experto. Al tiempo, como muestran algunos estudios (Yearley, 2002), la ciencia es adoptada por las organizaciones ecologistas en su labor de presión sobre otros actores políticos, pues les proporciona una mayor fuerza, efectividad y respeto por su labor.

A pesar de las consecuencias indeseadas en la naturaleza de la aplicación de la ciencia y la tecnología a partir de la industrialización, se sigue pensando que la ciencia ofrece el mejor conocimiento sobre los problemas ambientales. La ciencia proporciona evidencias de la ocurrencia de los daños sobre el medio y ofrece respuestas y soluciones a los mismos (Yearley, 2000). Sin embargo, se acepta cada vez más que el conocimiento científico tiene debilidades. Debilidades 
internas, referidas a los propios métodos de producción y validación del conocimiento científico, tal y como pone de manifiesto la teoría de la ciencia y la sociología del conocimiento científico; y debilidades externas, fruto de la compleja relación de la ciencia, en tanto que institución, con otros actores e instituciones sociales y del aumento de los riesgos producidos por la misma.

El creciente uso del conocimiento científico por parte de los políticos en la implementación de políticas públicas deviene en una paradoja en la relación entre ciencia y política (Weingart, 1999). El uso inflacionario del conocimiento científico por parte de los políticos produce una cientificación de la política, pero, también, una politización de la ciencia. El incremento en el uso del consejo científico no incrementa el nivel de certeza de las políticas basadas en este consejo. El conocimiento científico está sometido a incertidumbre y los problemas medioambientales, como el cambio climático o la erosión del suelo, plantean controversias que escapan al ámbito del conocimiento. Los políticos usan este conocimiento como legitimador de sus acciones, dado el amplio prestigio social de la ciencia, pero esta utilización creciente produce una deslegitimación de la política y, también, del mundo científico.

En el ámbito del medio ambiente y de las políticas ecológicas, las limitaciones de la ciencia en la solución de los problemas medioambientales se hacen más patentes. Las controversias sobre este tema plantean cuestiones de orden moral y político que no pueden ser resueltas simplemente por la investigación científica (Yearley, 2000). El medio ambiente es un sistema complejo, en el que predomina la incertidumbre y no existe una única perspectiva de análisis. Sus problemas tienen más de una respuesta o, en ocasiones, no poseen respuesta. Por este motivo, los valores desempeñan un papel determinante en el proceso de toma de decisiones sobre la gestión del medio ambiente (Funtowicz y Ravetz, 1999). La búsqueda de la verdad no es el único objetivo a perseguir de la aplicación del conocimiento científico, sino mejorar la calidad del proceso político de toma de decisiones.

En este contexto de ambivalencia en la relación entre la institución de la ciencia y la sociedad, dos perspectivas teóricas de la sociología intentan explicar, entre otros aspectos, el papel de la ciencia y la tecnología en la crisis medioambiental y en las transformaciones sociales derivadas de ella. Ambas teorías, la teoría de la sociedad del riesgo tal y como ha sido formulada por Beck (1998, 2002) y la modernización ecológica, han despertado un gran interés entre los estudios de sociología del medio ambiente. Las relaciones entre ambas perspectivas, sus similitudes y diferencias también han recibido la atención de distintos estudios (Mol y Spaargaren, 1993; Cohen, 1997; Buttel, 2000). La idea central al comparar ambas perspectivas es, como afirman Mol y Spaargaren, que "por su tono general pesimista y por su cuestionamiento del papel de la ciencia y la tecnología en la superación de una catástrofe 0ecológica, la teoría de la sociedad del riesgo parece fundamentalmente contradecir la teoría de la modernización ecológica» (Mol y Spaargaren, 1993, p. 431). Ambas teorías difieren en el papel asignado a la ciencia en la superación de la crisis ecológica. La relación entre sociedad y medio ambiente y el proceso de cambio social 
derivado de la crisis ambiental en la modernidad tardía es enfocado de modo diferente por la teoría de la sociedad del riesgo y la modernización ecológica. Sin embargo, ambas perspectivas resultan complementarias en numerosos aspectos. La comparación entre ambas puede proporcionar una mejor comprensión del papel de la ciencia en la crisis medioambiental, así como sugerir reflexiones sobre el futuro de la ciencia en la sociedad.

\section{El papel de la ciencia en la teoría de la sociedad del riesgo}

El concepto de riesgo ha alcanzado una importancia significativa en numerosas disciplinas científicas y, especialmente, en las ciencias sociales. Desde su origen como concepto empleado en la economía para hacer referencia al riesgo en la obtención de beneficio (Luhmann, 1996), el término se ha convertido en un elemento clave en el estudio de los problemas sociales derivados de la sociedad moderna. $\mathrm{Al}$ análisis del riesgo se han acercado distintas aproximaciones teóricas, de carácter psicológico unas, antropológico y sociológico otras, en un intento de dar cuenta de las distintas dimensiones del riesgo en la sociedad (Dietz, Frey y Rosa, 1992). Pero, fundamentalmente, ha sido el sociólogo alemán Ulrich Beck, a través de su obra publicada en 1986 Risikogesellschaft. Auf dem Weg in eine andere Moderne, quien ha contribuido al desarrollo de una teoría de la sociedad del riesgo en sociología. Desde entonces, la teoría de la sociedad del riesgo se ha convertido en una perspectiva muy influyente en el pensamiento sociológico y social de principios del siglo XXI, tanto por su planteamiento teórico en el análisis de la sociedad moderna, como por su visión, para algunos apocalíptica, del futuro de la sociedad

En la obra de Beck $(1998,2002)$ es posible distinguir dos conceptos fundamentales que sintetizan la perspectiva teórica del autor: el concepto de sociedad del riesgo y el de modernidad reflexiva, ambos estrechamente relacionados. El argumento básico de la teoría de la sociedad del riesgo es que en el seno de la modernidad se produce un proceso de cambio social por el que la sociedad industrial evoluciona hacia la sociedad del riesgo, en la que la producción de riesgos y la individualización se convierten en los procesos sociales predominantes. Se argumenta que la modernidad entra, así, en una nueva fase caracterizada por su reflexividad. El proceso de modernización se torna, desde esta óptica, reflexivo, es decir, las propias instituciones sobre las que se ha asentado son cuestionadas, repensadas en esta nueva fase de la modernidad.

Una idea esencial de la teoría desarrollada por Beck es que en la sociedad del riesgo, cuyos inicios se podrían establecer en el último cuarto del siglo XX, la lógica de la producción y el reparto de los riesgos sustituye a la lógica de la producción de riqueza, dominante en la sociedad industrial. Si en la sociedad industrial o de clases el paradigma de la desigualdad social consistía en el reparto y la distribución de la riqueza socialmente producida, el paradigma principal en la sociedad del riesgo es el reparto, la minimización, la canalización de los riesgos generados por el desarrollo económico y científico técnico del proceso modernizador, nos dice el autor. La aparición de los riesgos responde al 
propio proceso modernizador, que en su propio desarrollo amenaza con la destrucción de las bases sobre las que se ha asentado. Los riesgos son daños irreversibles que, producidos socialmente, poseen la capacidad de destruir la vida misma sobre la Tierra. La civilización se pone en peligro a sí misma. La existencia de peligros en la vida de los individuos es un fenómeno que ha existido a lo largo de la historia. Pero los riesgos, explica el sociólogo alemán, se distinguen de los peligros que tradicionalmente han alertado la vida de los individuos por su carácter global y por el origen de sus causas, ahora en la propia modernidad.

Los riesgos que amenazan a la sociedad moderna son amenazas globales; no entienden de estados ni de clases sociales. Como afirma Beck, aunque el reparto de los riesgos siga la lógica de la desigualdad de clases, de modo que las clases bajas sufren más los riesgos, la lógica de este reparto es esencialmente diferente, pues tarde o temprano el riesgo se torna hacia quien lo produce. Nadie está seguro ante los nuevos riesgos, que, tanto si se tratan del cambio climático como del reparto de sustancias tóxicas en el aire, los alimentos o el agua, extienden su amenaza a todo el planeta. Por lo tanto, una característica fundamental de los nuevos riesgos es, desde el punto de vista de esta teoría, su carácter global, supranacional y no específico de una sola clase social.

En segundo lugar, los riesgos son generados por la modernidad. La teoría de la sociedad del riesgo considera que el proceso de modernización tiene la semilla de su autodestrucción, pues «la sociedad del riesgo es una sociedad catastrófica» (Beck, 1998, p. 30). Los riesgos son generados por la modernización, es decir, tienen un carácter antrópico, pues es el hombre quien, con el desarrollo de la técnica y de la ciencia modernas, así como a través del crecimiento económico, crea los riesgos. La sociedad del riesgo se distingue de otras etapas históricas por ser el desarrollo de sus mismas instituciones las que ponen en peligro la continuidad de la misma. He aquí una de las ideas centrales de la teoría.

Beck introduce un elemento analítico fundamental con el concepto de reflexividad. Desde su punto de vista, al reflexionar y tratar de hacer frente a los riesgos, el proceso de modernización se vuelve reflexivo (Beck, 1998). La reflexividad caracteriza a esta nueva fase de la modernidad. Se considera que la modernidad atraviesa un periodo de crisis caracterizado por la autotransformación de la misma, que conduce hacia una nueva modernidad. El concepto de «modernidad reflexiva» es un concepto esencial en la obra de Beck y, como afirman algunos autores (Spaargaren, 2000), mucho más central para el análisis sociológico del cambio social frente a la crisis ecológica que el concepto de «sociedad del riesgo».

La idea esencial desarrollada por Beck es que el carácter reflexivo de la modernidad tardía supone un cuestionamiento de las instituciones en las que se ha basado la modernidad. Algunos de los pilares básicos del proceso modernizador, como la ciencia, el progreso o el industrialismo, son puestos en duda en esta fase de la modernidad. Los riesgos, generados por el mismo proceso modernizador, son los elementos que favorecen el cambio, pues confrontan 
a la modernidad consigo misma. Las consecuencias no deseadas de la modernidad, nos dice Beck, producen una autorreflexión sobre las causas y la gestión de las mismas que llevan a la modernidad hacia la modernidad reflexiva. Ésta es una idea central en la teoría de la sociedad del riesgo y la modernidad reflexiva, que la separa ampliamente de la teoría de la modernización ecológica.

La teoría de la sociedad del riesgo y la modernidad reflexiva presta especial atención al papel de la ciencia en la sociedad del riesgo (Goldblatt, 1996). Una de las instituciones que es afectada especialmente por el proceso modernizador reflexivo es la ciencia. Desde este enfoque, son numerosos los aspectos tratados en relación con la institución de la ciencia en la sociedad del riesgo. En términos generales, la visión de la ciencia y la tecnología se puede considerar negativa, pues la teoría de la sociedad del riesgo apunta, básicamente, al desarrollo científico-técnico como el causante principal de los riesgos que emergen de modo irreversible del proceso modernizador. En la perspectiva sobre la institución de la ciencia, la teoría de la sociedad del riesgo y la teoría de la modernización ecológica parecen contraponerse, pues ambas centran su enfoque en aspectos distintos de la realidad social.

La ciencia y la tecnología son analizadas ampliamente en la obra de Beck. La idea central sobre el papel de la ciencia en las transformaciones institucionales de la modernidad tardía se pueden resumir en la idea según la cual la ciencia se «ha convertido en (con) causa, instrumento de definición y fuente de solución de riesgos» (Beck, 1998, p. 203). Esta triple caracterización de la ciencia resume todos los aspectos tratados en la teoría de la sociedad del riesgo y la modernización reflexiva. La ciencia es, así, causa de los riesgos que amenazan con la destrucción del planeta, al tener muchos de ellos origen en el desarrollo tecnológico. Chernobyl se convierte, aquí, en el ejemplo paradigmático de la tragedia en la sociedad del riesgo, en una especie de conmoción para la humanidad que hace cambiar la percepción de los desarrollos tecnológicos por parte de la población de las sociedades avanzadas. Pero la ciencia es, a su vez, argumenta el autor, definidora de los riesgos, pues éstos son construidos social y científicamente. Y es, en último lugar, la solución a los problemas que ella misma genera, aspecto que Beck considera que da lugar a una ampliación del ámbito de la cientificación.

En la visión de la teoría de la sociedad del riesgo, la ciencia y la tecnología están en el origen de gran parte de los riesgos que amenazan el futuro de la humanidad. El proceso de modernización se enfrenta con consecuencias no deseadas generadas por este mismo proceso, lo que provoca la autoconfrontación de la modernidad. La modernidad se hace reflexiva y la institución de la ciencia es cuestionada por la sociedad y por la misma ciencia, pues, tal y como afirma Beck, la crítica a la ciencia también es científica, de modo que la ciencia critica a la ciencia misma. La ciencia, nos dice el autor, es desmitificada en la sociedad del riesgo; baja de su pedestal al que los logros del progreso tecnológico le llevaron y pierde la verdad. La desmitificación de la ciencia se produce, según Beck, en el ámbito interno y externo, es decir, en el mismo proceso 
de validación del conocimiento científico y en su relación con otras esferas de la sociedad. En el ámbito interno, la teoría de la ciencia, la sociología del conocimiento científico o los estudios etnográficos sobre la actividad científica muestran los procesos sociales que subyacen a la producción de conocimiento científico, lo que debilita las bases del universalismo científico (Beck, 1998, p. 213). En el ámbito externo, dice Beck, la ciencia se convierte en el principal responsable de los desastres ecológicos, en un elemento generador de riesgos. Los peligros derivados de la energía nuclear, la contaminación del medio ambiente, la difusión de sustancias venenosas en el agua o los alimentos, entre otros fenómenos, sitúan a la ciencia y la tecnología ante la opinión pública en el origen de los riesgos.

A la vez que la ciencia contribuye a la producción de riesgos, la teoría de la sociedad del riesgo subraya el papel determinante de la ciencia en la definición y la construcción social de los riesgos. La teoría de la sociedad del riesgo ha adoptado, fundamentalmente, una perspectiva constructivista sobre los problemas medioambientales. Bien es cierto que Beck (2002) distingue ambas aproximaciones dentro de su teoría, la realista, según la cual los peligros existen y son globales, y la constructivista, según la cual los peligros son construidos socialmente. El proceso de construcción social de los riesgos es un proceso complejo en el que intervienen distintos actores sociales. En este proceso desarrolla un papel determinante el conocimiento público, lo que la gente conoce y percibe de los peligros que le rodean y, sobre todo, el conocimiento experto. El conocimiento y el material científico se convierten en elementos esenciales en la construcción social de los riesgos. «Los peligros ecológicos no existen en sí mismos, sino que se definen, ocultan o dramatizan estratégicamente en la esfera pública con la ayuda de material científico suministrado a tal efecto» (Beck, 2002, p. 34).

Afirma Beck que en la construcción científica de los riesgos prima la racionalidad científica, en ocasiones enfrentada a la racionalidad social. La racionalidad científica define lo que son riesgos, establece la cantidad de ellos que se pueden asumir, la probabilidad de ocurrencia que hay. La ciencia fija los riesgos, pero la población los percibe. Los científicos y los expertos se convierten en los depositarios del monopolio de la definición y diagnóstico de los riesgos (Beck, 2003). Tan sólo el conocimiento científico sirve como legitimador de los peligros, sólo a través de él se puede establecer los que son asumibles, aquéllos que pueden ser considerados como no riesgo. Sin embargo, explica Beck, este monopolio de científicos e ingenieros está siendo cuestionado por la sociedad, pues el papel de la institución de la ciencia, ante la magnitud de los riesgos, es puesto en tela de juicio en la modernidad reflexiva.

Desde la perspectiva de la sociedad del riesgo, la modernidad produce una ciencia responsable de la producción de riesgos, pero, también, de la solución de los mismos. El monopolio en el diagnóstico de los riesgos que la sociedad concede a la ciencia se traduce en un monopolio en la solución de los mismos. Sólo el conocimiento científico es capaz de aportar soluciones a los peligros ecológicos, por lo general de naturaleza compleja. Sin embargo, esa misma 
naturaleza compleja de los peligros ecológicos determina la existencia de incertidumbre científica, cada vez más reconocida públicamente. En opinión de Beck, no existen soluciones de expertos sobre el riesgo, pues nunca el conocimiento científico es capaz de evaluar las soluciones culturalmente aceptables. La racionalidad científica se vuelve vacía sin racionalidad social, pero la racionalidad social es ciega sin racionalidad científica, nos dice el autor.

En último lugar, la ciencia, además de ser causa, elemento de definición y fuente de solución de los riesgos, es considerada, en la teoría de la sociedad del riesgo y la modernidad reflexiva, como elemento central en la crítica reflexiva sobre la ciencia y la tecnología. La ciencia, y especialmente las ciencias sociales, vuelven su mirada sobre la actividad científica y sobre el papel de la ciencia en la producción y la gestión de los peligros de un modo crítico. Según Beck, la crítica de la ciencia se cientifica, como lo demuestra el uso de datos y argumentos científicos por parte del movimiento ecologista. Los distintos actores sociales, sea la industria, el gobierno, los ciudadanos o el movimiento ecologista, utilizan el conocimiento científico para legitimar sus posiciones, en muchas ocasiones enfrentadas.

Como afirman Mol y Spaargaren (1993), la teoría de la sociedad del riesgo parece ver la causa de todos los problemas medioambientales en la ciencia y la tecnología, en vez de hacerlo en las relaciones sociales en que éstas se aplican. Esta aproximación centrada excesivamente en la ciencia da lugar a una visión escéptica y negativa del papel de la ciencia en la solución de los problemas medioambientales. Esta visión ha condicionado las propuestas realizadas por la teoría de la sociedad del riesgo para avanzar hacia la sociedad sostenible. Así, autores como Mol y Spaargaren (1993) han criticado las propuestas dirigidas por la teoría de la sociedad del riesgo hacia una organización interna diferente de la ciencia y un método científico diferente. Se consideran propuestas innecesarias para la solución de los problemas medioambientales.

En una de las últimas obras de Beck (2002), las propuestas de cambio en la relación entre ciencia y sociedad se han dirigido a otros aspectos de la misma. Como muestra la teoría de la sociedad del riesgo, a fines del siglo XX la incertidumbre científica y la falibilidad de los modelos científicos empleados en la gestión de los problemas medioambientales es reconocida públicamente. Esto abre, según Beck, el espacio a la democratización de la relación entre ciencia y política. La tecnocracia autoritaria debe, según las propuestas del autor de la teoría de la sociedad del riesgo, dejar paso a una tecnocracia crítica, consciente de sus limitaciones y abierta al escrutinio público (Beck, 2002). La ciencia debe abrirse desde dentro a la opinión pública, mostrar sus limitaciones y dejar que su praxis se contraste públicamente. Solo así, dice el autor, se abrirá el camino hacia la "democracia ecológica» (Beck, 2003) que aleje a la sociedad de la tiranía de la tecnología. 


\section{La ciencia en la perspectiva de la modernización ecológica}

Junto a la teoría de la sociedad del riesgo, la denominada «teoría de la modernización ecológica», desarrollada a partir de los años ochenta, se ha convertido en una de las perspectivas teóricas de mayor relevancia entre la sociología medioambiental de los últimos años, de manera que ha alcanzado en la actualidad un cuerpo importante de desarrollos teóricos y empíricos. El concepto de modernización ecológica fue empleado, inicialmente, para designar un estilo, una vía de actuación en las políticas medioambientales de algunos países europeos durante los años ochenta. Frente al fallo de la política medioambiental del estado y frente a un movimiento ambiental radicalizado y anclado en posturas ideológicas cercanas al desindustrialismo, la modernización ecológica se erige como una tercera vía hacia la reforma medioambiental de la sociedad (Mol, 1999). En este contexto de cambio en las políticas del medio ambiente, durante los años ochenta surge la teoría de la modernización ecológica, como un esfuerzo teórico para analizar el proceso de cambio en las instituciones sociales frente a los desafíos medioambientales.

En los estudios sobre los orígenes de la teoría de la modernización ecológica (Mol y Spaargaren, 1993; Mol, 1999), se suelen considerar los trabajos de Joseph Huber y Martín Jänicke a mediados de los años ochenta como los inauguradores de esta corriente teórica. Los elementos centrales tratados en estos primeros trabajos son el papel de la innovación tecnológica en la reforma ecológica de la producción, el creciente protagonismo de los actores económicos en el camino hacia la sostenibilidad y las transformaciones en la política medioambiental y en el papel del estado que tienen lugar en algunos países europeos. Estos primeros análisis originados en Alemania trataban de dar cuenta de los cambios en las prácticas, los discursos y las instituciones medioambientales que estaban teniendo lugar en estos años en algunas sociedades europeas. Su enfoque era, fundamentalmente, sistémico, evolucionista y estaba centrado en el ámbito estatal. Desde entonces, la teoría de la modernización ecológica ha ido ampliando sus dimensiones de análisis, así como sus ámbitos de estudio, abordando los distintos procesos de cambio en las instituciones sociales de las sociedades. Así, se ha convertido en una teoría del cambio social que intenta describir y analizar «las transformaciones de las sociedades industriales contemporáneas frente a los desafíos ecológicos» (Mol, 2002). Junto al carácter analítico de la teoría de la modernización ecológica, también se ha mantenido cierto tono normativo o prescriptivo, en el sentido de un conjunto de pautas deseables para avanzar hacia la sociedad sostenible, aspecto, éste último, que no se debe confundir con la dimensión teórica.

Durante los últimos años, la perspectiva de la modernización ecológica ha avanzado en sus esfuerzos teóricos y empíricos por comprender la transformación medioambiental de la sociedad, analizando el cambio en las distintas esferas económica, política y cultural. Desde una óptica inicial más centrada en el análisis de la reforma ecológica de la producción, en la línea de los trabajos de Huber (véase Huber, 2000), se ha analizado el proceso de modernización 
política, los cambios en las pautas de consumo y los estilos de vida o los cambios culturales como partes de un proceso más general de cambio social. Fundamentalmente, han sido Mol y Spaargaren (Mol y Spaargaren, 1993; Mol, 1999, 2002; Spaargaren, 2000; Mol y Sonnenfeld, 2000) quienes han expuesto, de un modo más sistemático, la perspectiva analítica de la modernización ecológica, al tiempo que han contribuido al desarrollo teórico y empírico de la misma. De un modo abstracto, Mol y Spaargaren (Mol y Spaargaren, 1993) identifican un proceso de creciente independencia de la esfera ecológica frente a otras esferas de la sociedad. La preocupación por el medio ambiente es institucionalizada e incorporada a las prácticas sociales. La racionalidad ecológica se introduce en el funcionamiento de las instituciones sociales, con lo que adquiere una entidad propia frente a la racionalidad económica o política. El objetivo de la teoría de la modernización ecológica es, por tanto, analizar y explicar las transformaciones sociales inducidas por el medio ambiente en las sociedades avanzadas.

Las transformaciones sociales centrales que han sido analizadas desde la perspectiva de la modernización ecológica han sido agrupadas en cinco procesos (Mol y Sonnenfeld, 2000): a) cambio en el papel de la ciencia y la tecnología ante los problemas ambientales; b) creciente importancia de las dinámicas de mercado y de los agentes económicos en la reforma ecológica; c) transformación del papel del estado en la política del medio ambiente; d) cambios en la posición, el papel y la ideología de los movimientos medioambientales; e) aparición de un nuevo discurso medioambiental. Básicamente, es posible distinguir, a modo de esquema conceptual, cambios en tres esferas sociales: económica, a través de la reforma ecológica de la producción y el consumo; política, proceso de modernización política e incremento de la colaboración entre actores sociales, y cultural, a través del cambio en el papel de la ciencia y la tecnología y la aparición de una capacidad cultural para la modernización ecológica.

Pese a que la ciencia y la tecnología han ocupado un papel fundamental en la perspectiva de la modernización ecológica, se ha prestado escasa atención al papel de la ciencia en la sociedad. La institución de la ciencia ha sido analizada, desde esta perspectiva, dando una especial importancia a la producción de tecnología ecológica y su papel en la transformación de los procesos de producción y consumo. Los estudios de la modernización ecológica han prestado especial atención al papel de la ciencia en tanto que cuerpo de conocimientos expertos sobre los problemas medioambientales y en tanto que creadora de innovación y tecnología ambiental. Tal y como afirma Mol (2000), el cambio en la institución de la ciencia y la tecnología es una de las dimensiones básicas de la perspectiva de la modernización ecológica, junto con el estudio de la economía, el estado y los movimientos sociales. La idea central es que la ciencia y la tecnología tienen un papel importante en la prevención y en la solución de los problemas medioambientales, y no sólo en su creación. Las opciones reactivas frente a los problemas del medio ambiente son sustituidas por aproximaciones sociotecnológicas preventivas, de modo que la preocupa- 
ción por el medio ambiente es incorporada en todas las fases de la innovación, tanto técnica como organizativa (Mol, 2000). La ciencia medioambiental se convierte, así, desde esta perspectiva, en el elemento esencial en el proceso de aprendizaje institucional que supone la modernización ecológica al permitir identificar estrategias para integrar las «externalidades» ecológicas en las prácticas de la sociedad industrial (Hunold y Dryzek, 2001). Sin embargo, la perspectiva de la modernización ecológica ha olvidado numerosos aspectos de la institución científica considerada en un sentido amplio, es decir, como comunidad científica, como organización en relación con otras instituciones y como conjunto de conocimientos, teorías y descubrimientos.

La centralidad de la ciencia y la tecnología en la teoría de la modernización ecológica es ya patente en la obra de Huber sobre las transformaciones en la producción industrial en las sociedades avanzadas. En la obra de Huber (véase un trabajo más reciente en Huber, 2000), la tecnología moderna es considerada como el elemento clave en la reorganización institucional de la sociedad moderna para hacer frente a la crisis ecológica. La economización de la ecología con mecanismos como las ecotasas o la valoración de los recursos naturales conduce a una ecologización de la economía que produce el cambio en la organización de los procesos de producción y consumo en la sociedad, que, a su vez, se traduce en la transformación ecológica de la sociedad. El proceso de reconstrucción de la sociedad es un proceso de progresiva modernización facilitado por la tecnología (Huber, en Mol y Spaargaren, 1993).

Como afirma Spaargaren (2000b), la prominencia que la teoría de la modernización ecológica concede a la ciencia y a la tecnología en el cambio hacia la sociedad sostenible ha sido, en ocasiones, mal interpretada. Así, entre las críticas que se han realizado a la modernización ecológica en su dimensión programática, está la de una excesiva confianza en la ciencia y la tecnología para la solución de los problemas ambientales y el carácter tecnocrático de esta perspectiva, al reducir los problemas del medio a problemas de carácter técnico. En este sentido, algunos autores (Cherni, 2003) critican la falta de radicalidad de la modernización ecológica al proponer un crecimiento ecologizado, a través de la introducción de innovaciones tecnológicas y organizativas, como solución a la crisis ambiental.

Desde la perspectiva de la modernización ecológica, la tecnología desarrolla un papel esencial en la reforma de los procesos productivos y de consumo. La innovación, la difusión de nuevas tecnologías o técnicas de gestión en las organizaciones industriales, es analizada como el elemento básico en la transformación de la producción. El concepto de industria ecológica (Huber, 2000), cada vez con mayor difusión entre los estudios de modernización ecológica de la producción, destaca, también, el papel de la innovación tecnológica en el cambio hacia una sociedad sostenible. Este concepto se refiere a un metabolismo industrial consistente con el metabolismo de la naturaleza. A través de la estrategia de consistencia, frente a las estrategias tradicionales de suficiencia o eficiencia, la industria ecológica permitiría avanzar hacia una sociedad sostenible. En la base de la industria ecológica está la transformación de las estructuras 
industriales tradicionales a través de innovaciones tecnológicas esenciales, estructurales. La tecnología tiene, aquí, un papel esencial, que no se limita al incremento de la eficiencia de los procesos productivos. En ciertos círculos, la estrategia de modernización ecológica es asimilada, erróneamente, a una «revolución de la eficiencia», es decir, un incremento de la producción con menor uso de materia y energía (Spaargaren, 2000a). Con el concepto de industria ecológica, la tecnología es vista como introductora de cambios radicales en el metabolismo industrial, es decir, en los flujos de uso de la energía y los materiales, de tal modo que sea consistente con el metabolismo de la naturaleza.

La centralidad de la ciencia y la tecnología en la reforma medioambiental de la sociedad plantea numerosos retos para los actores económicos, fundamentalmente para la industria, así como para el mundo de la investigación y para la política ambiental. El papel de la ciencia cambia en la moderna política ambiental (Lundqvist, 2000). La investigación en medio ambiente se incrementa y amplía sus horizontes. De una investigación orientada al descubrimiento del problema medioambiental y al análisis de causa y efecto, se avanza hacia una investigación orientada a la solución de problemas. El elemento esencial en la búsqueda de soluciones a los nuevos problemas ambientales es la promoción de tecnologías ecológicas. Por este motivo, la ciencia y la tecnología ocupan un lugar especial en la nueva política ambiental, necesitada de nuevos recursos para hacer frente a la crisis ambiental. Los análisis empíricos sobre el proceso de modernización ecológica en las sociedades europeas revelan este cambio en el papel de la ciencia y la tecnología. En un análisis sobre el proceso de modernización ecológica en Suecia (Lundqvist, 2000), se constata que la promoción de la ciencia y la creación de tecnologías ecológicas se convierte en un elemento clave de la política ambiental del gobierno a partir de los años noventa. Se produce una nueva organización en la investigación medioambiental, con la creación de nuevas agencias, fundaciones e institutos de investigación estratégica en medio ambiente.

En definitiva, la perspectiva de la modernización ecológica se ha centrado en el nuevo papel que la ciencia y la tecnología adquieren en la trasformación de las sociedades avanzadas frente a los retos medioambientales. Así, se ha incidido en el papel de la ciencia en la creación de innovaciones tecnológicas que permiten la prevención y también la solución de los problemas medioambientales. Sin embargo, como ha afirmado Spaargaren (2000b), autor que ha contribuido al desarrollo de la teoría de la modernización ecológica, numerosos aspectos de la relación entre la institución de la ciencia y la sociedad han sido olvidados en el cuerpo de estudios de la modernización ecológica. Se ha prestado muy poca atención al papel de los científicos en el proceso de modernización ecológica, a la relación entre expertos y público o a la relación entre expertos y políticos. La solución propuesta por Spaargaren es incorporar aspectos analíticos de la teoría de la modernización reflexiva a la teoría de la modernización ecológica, de modo que se puedan subsanar las debilidades teóricas de la modernización ecológica. Pero la disparidad entre ambos enfoques hace difícil tal adaptación de ambas perspectivas. 
Pero la teoría de la modernización ecológica debe profundizar, desde nuestra perspectiva, en el conocimiento de las transformaciones en el papel de la ciencia y la tecnología en el contexto de la reforma medioambiental de las sociedades avanzadas. La trayectoria de investigación iniciada por autores como Huber o Jänicke sobre el papel de la tecnología en la modernización ecológica debe ampliarse a otras esferas de análisis. Para ello, se considera muy interesante la incorporación a la teoría de la modernización ecológica del concepto de post-normal science (Funtowicz y Ravetz, 1999; Ravetz, 2003). Básicamente, este concepto se refiere a un nuevo tipo de ciencia adaptada al nuevo contexto de relaciones entre sociedad y medio ambiente. Según estos autores, el modelo de interacción entre ciencia y política en el que los científicos dan un conocimiento básico para la toma de decisiones políticas es inadecuado en el contexto de la política del medio ambiente actual. La política del medio ambiente se desarrolla en un contexto de complejidad, tanto social como medioambiental. El medio ambiente es un sistema complejo y, por tanto, está sometido a incertidumbre. Ante esta incertidumbre, no existe una única perspectiva de aproximación. Los problemas medioambientales tienen más de una respuesta o no tienen respuesta, por lo que los valores desempeñan un papel muy importante. Según estos autores, en contextos de complejidad, hay un nuevo papel para la ciencia. La ciencia sigue siendo esencial para la solución de los problemas medioambientales, pero en un contexto de incertidumbre de los sistemas naturales y de relevancia de los valores humanos.

\section{Conclusiones}

La confrontación de dos perspectivas teóricas, la teoría de la sociedad del riesgo y la teoría de la modernización ecológica, permite ahondar en el conocimiento del papel de la ciencia en la sociedad y la cultura modernas. Ambas teorías dirigen su mirada, fundamentalmente, a las transformaciones institucionales en las sociedades industriales avanzadas derivadas de la crisis medioambiental. La interpretación de la crisis difiere sustancialmente en ambas perspectivas, que sí que coinciden en la importancia concedida a las cuestiones medioambientales, así como en la ubicación temporal de este fenómeno. Pero ambas perspectivas coinciden, también, en un aspecto que se ha considerado de vital importancia en este trabajo, como es la relevancia atribuida a la ciencia en las transformaciones de la sociedad al inicio del nuevo milenio.

Consideradas las similitudes entre ambos enfoques teóricos, la visión de la sociedad del riesgo y la perspectiva de la modernización ecológica tienen notables diferencias. Ambas perspectivas poseen enfoques distintos, dirigen su atención hacia fenómenos sociales diferentes, realizan un diagnóstico, un pronóstico y una terapéutica de la crisis muy diferente, lo que hace difícil conjugar ambas perspectivas. Sin embargo, de ambas perspectivas es posible extraer numerosos elementos analíticos de gran interés en el estudio de la ciencia en la sociedad y la cultura contemporáneas. En alguna medida, las dos perspectivas se complementan, pero la unificación de ambas en una 
misma línea de investigación puede confundir y dificultar el desarrollo teórico de cada enfoque.

La teoría de la sociedad del riesgo y la modernidad reflexiva, tal y como ha sido planteada por Beck, posee una mirada no sólo escéptica, sino también negativa sobre el papel de la ciencia y la tecnología en las sociedades avanzadas. La ciencia es causa, en gran medida, de los riesgos generados en el seno de la modernidad. Pero es, también, responsable de la definición de los riesgos y monopoliza la solución de los mismos al tiempo que se torna reflexiva y analiza y critica a la misma institución de la ciencia. En gran medida, las predicciones de la teoría son apocalípticas y plantean una visión negativa de la capacidad de la sociedad moderna para hacer frente a los problemas medioambientales. A pesar de este elemento negativo, el concepto de tecnocracia crítica y democratización en la relación entre ciencia y política abre las puertas a una sociedad gobernada por la ciencia, que no dominada por la ciencia.

La teoría de la modernización ecológica, con un enfoque distinto, centra su análisis en la reforma medioambiental de la sociedad. El análisis del cambio en el papel desempeñado por la ciencia y la tecnología en las sociedades avanzadas es central en la teoría de la modernización ecológica. La visión de los estudios enmarcados en la modernización ecológica es optimista y constructiva en cuanto al papel de la ciencia y la tecnología en la solución de los problemas medioambientales, puesto que concede especial importancia al papel de la innovación tecnológica y organizativa. En este sentido, muchos otros aspectos de la relación entre ciencia y sociedad han sido ignorados en esta perspectiva, pero la ciencia ha sido considerada como una pieza más en un conjunto teórico interesante, sugerente empíricamente y en mayor o menor medida codificado.

Es difícil establecer una única explicación sobre el papel que la ciencia y la tecnología han desempeñado y desempeñarán en el modo como las sociedades avanzadas hagan frente a los riesgos ecológicos. La reflexión desde ambas perspectivas teóricas permite elaborar algunas tesis fundamentales. En primer lugar, se hace patente que el mismo desarrollo científico-técnico que, ligado a un crecimiento económico sin precedentes, conduce a la sociedad industrial hacia un equilibrio inestable con su ecosistema, desencadena un proceso modernizador de nuevo cuño, caracterizado por su dimensión ecológica. Resulta difícil negar que la sociedad industrial ha generado numerosos riesgos sobre la salud de las personas y del planeta. Pero, del mismo modo, es equivocado despreciar el proceso modernizador y de reforma que tiene lugar en las sociedades avanzadas en los últimos años. Un proceso que conduce, a través de la activación de la sociedad, del esfuerzo y la colaboración entre los distintos actores sociales y de la aplicación efectiva de conocimiento científico y tecnológico, a mejoras progresivas en la calidad medioambiental de las sociedades.

La sociedad moderna se enfrenta, en los inicios del siglo XXI, a una bifurcación en su avance tras la crisis medioambiental. Las teorías analizadas anteriormente dan buena cuenta de esta dualidad. Se puede evolucionar hacia la 
sociedad del riesgo, con consecuencias dramáticas para la sociedad mundial y el ecosistema. Pero se puede, también, profundizar en el proceso modernizador, ahora en su vertiente ecológica, y avanzar hacia la reforma medioambiental de los procesos productivos y de consumo, del papel de la ciencia y la tecnología, de la política medioambiental y de la acción de los actores sociales, de modo que se consiga una mejor actuación ecológica de las sociedades avanzadas. No cabe ninguna duda que en esta reforma medioambiental la ciencia y la tecnología están llamadas a jugar un papel esencial.

Una segunda tesis, con grandes implicaciones para la investigación sociológica, se puede extraer del análisis de la teoría de la sociedad del riesgo y la modernización ecológica. El papel de la ciencia y la tecnología en la solución de los problemas medioambientales estará limitado o potenciado por el modo como se institucionalice el proceso político del medio ambiente. De la relación entre expertos, políticos, organizaciones y público y de la creación de confianza entre los ciudadanos en la capacidad de las instituciones modernas de dar respuesta a los riesgos ecológicos dependerán la calidad del proceso modernizador y la consecución de una democracia ecológica legítima.

En definitiva, de la confrontación entre la teoría de la sociedad del riesgo y la modernización ecológica es posible aprender numerosos aspectos sobre la ciencia y la tecnología y sobre su papel en la adaptación de las sociedades modernas a los retos planteados por los desafíos ecológicos. La institución de la ciencia ha sido un elemento central en el desarrollo de la sociedad moderna. Es difícil imaginar un futuro en el que la actividad científica no desempeñe un papel central en el terreno económico, político y cultural de las sociedades. Pero la ciencia y la tecnología no son elementos aislados, así como tampoco el único motor del cambio social. Ambas se encuentran insertas en un sistema de relaciones más amplio y complejo como es el sistema social, cambiante y dinámico. Por este motivo, es adecuado aproximarse a la comprensión de estos fenómenos sociales guiados por la luz de una perspectiva teórica como pueden ser la teoría de la modernización ecológica o la teoría de la sociedad del riesgo. Ambas se han mostrado como esfuerzos teóricos útiles y relevantes en el estudio de las relaciones entre ciencia, medio ambiente, cultura y sociedad. De su conocimiento puede depender, en gran medida, la sostenibilidad de la sociedad futura.

\section{Bibliografía}

BECK, U. (2003). «From Industrial Society to the Risk Society: Questions for Survival, Social Structure, and Ecological Enlightenment». En DrYZEK, J.; SCHLOSBERG, D. Debating the Earth: The Environmental Politics Reader. Oxford: Oxford University Press.

- (2002). La sociedad del riesgo global. Madrid: Siglo XXI.

- (1998). La sociedad del riesgo. Barcelona: Paidós.

ButTel, F. (2000). «Ecological modernization as social theory». Geoforum, vol. 31. CoHEn, M. (1997). «Risk society and ecological modernisation. Alternative visions for post-industrial nations». En Futures, vol. 29, núm. 2. 
Commoner, B. (1975) [1966]. Science and Survival. Versión en castellano: Ciencia y Supervivencia. Barcelona: Plaza y Janés.

DieTZ, T.; FreY, R. S.; Rosa, G. (2002). «Risk, Technlogy and Society». En DunlaP, R.; Michelson, W. (eds.). Handbook of Environmental Sociology. Westport: Greenwood Press.

FUNTOWICZ, S.; RAVETZ, J. (1999). «Post-normal science: environmental policy under conditions of complexity». <http://www.jvds.nl/pns/pns.htm>

GoldBlatt, D. (1996). Social Theory and the Environment. Cambridge: Polity Press. HubER, J. (2000). «Towards Industrial Ecology: Sustainable Development as a Concept of Ecological Modernization». Journal of Environmental Policy and Planning, núm. 2.

HunOlD, C.; DrYZEK, J. (2001). «Greening the state? Ecological Modernization between state and movement in the USA, UK, Germany and Norway». Joint Sessions of the European Consortium for Political Research, Grenoble.

Luhmann, N. «El concepto de riesgo». En Beriain, J. (coord.) (1996). Las consecuencias perversas de la modernidad: modernidad, contingencia y riesgo. Barcelona: Anthropos.

Martell, L. (1994). Ecology and Society. An introduction. Cambridge: Polity Press. Mol, A. (2002). «Modernización ecológica: Transformaciones industriales y reforma medioambiental». En REDCLIFT, M.; WoOdGATE, G. Sociología del medio ambiente. Una perspectiva internacional. Madrid: McGraw-Hill.

Mol, A.; SONNENFELD, DA. (eds.) (2000). Ecological Modernisation around the World: Perspectives and Critical Debates. Londres: Frank Cass.

MoL, A. P. J. (1999). «Ecological Modernization and the Environmental Transition of Europe: Between National Variations and Common Denominators». En Journal of Environmental Policy and Planning, núm. 1.

Mol, A. P. J.; SPAARGAREN, G. (1993). «Environment, modernity and the risk-society: the apocalyptic horizon of environmental reform». En International Sociology, vol. 4, núm. 8.

RAVETZ, J. (2003). «The post-normal science of precaution». Futures, XX.

SpaArgaren, G. (2000a). «Ecological Modernization Theory and Domestic Consumption». Journal of Environmental Policy and Planning, núm. 2.

- (2000b). «Ecological Modernization Theory and the Changing Discourse on Environment and Modernity». En SpaArgaren, G.; Mol, A. P. J.; Buttel, F. Environment and Global Modernity. Londres: Sage.

STEEL, B.; LIST, P.; LACH, D.; SHINDLER, B. (2004). «The role of scientists in the environment policy process: a case study from the American west». Environmental Science \& Policy, vol. 7.

WEINGART, P. (1999). «Scientific expertise and political accountability: paradoxes of science in politics». Science and Public Policy, vol. 26, núm. 3.

Yearley, S. (2002). «Ciencia y Medio Ambiente». En Redclift, M.; Woodgate, G. Sociología del medio ambiente. Una perspectiva internacional. Madrid: McGraw-Hill. 